\title{
On Demand Channel Assignment Method FOR CHANNEL DIVERSITY (ODCAM)
}

\author{
Sidi Ould CHEIKH \\ Nouackchott University, Nouackchott, Mauritania
}

\begin{abstract}
The IEEE 802.11s Wireless Mesh Networks (WMN) is a new multi-hop technology increasing the coverage of IEEE 802.11 Wireless Network and providing Internet access. In order to increase the mesh network capacity, the WMN has evolved from single-radio single-channel architecture to Multi-Channel MultiRadios (MC-MR) architecture. In MC-MR the main challenge of the WMN is the channel assignment. In this article, we propose a new channel assignment method based on channel diversity. This new method named ODCAM (On Demand channel Assignment Method for channel diversity) defines a channel diversity mechanism used to select a new channel along the path between the source and the destination. The best path between the source and the destination is provided by the HWMP (Hybrid Wireless Mesh Protocol) protocol using MWCETT (Modified Weighted Cumulative Expected Transmission Time) an extension of the WCETT metric. The simulation results show the ODCAM performance compared with an hybrid approach.
\end{abstract}

\section{KEYWORDS}

Wireless Mesh Network, IEEE 802.11s, Routing, Multi-channel Multi-radio.

\section{INTRODUCTION}

The IEEE 802.11s Wireless Mesh Networks (WMN) is a new technology increasing the coverage of IEEE 802.11 Wireless Network [1]. It is characterized by multi-hop topology [2], simplicity of deployment and providing low cost Internet connectivity. Three main approaches can be identified to implement mesh routing functionality, which differ in the protocol layer implementation (MAC, network or in-between layer).

The layer-2 approach provides frames forwarding and path selection at layer-2, the in-between layer (layer-2.5) approach uses an additionnal software layer interposed between layer-2 and layer-3. The layer-3 approach implements mesh functionality at network layer [3].

The WMN based-IEEE 802.11s task group proposed a layer-2 approach, which is a MAC layer routing protocol HWMP (Hybrid Wireless Mesh Protocol), based on MAC addresses [2].

The main challenge of the WMN-based IEEE 802.11s is how to increase the capacity to support a multi-hop QoS requirement, such as throughput and end-to-end delay [4]. In order to increase the mesh network capacity, the WMN has evolved from single-radio single-channel architecture to Multi-Channel Multi-Radios (MR-MC) architecture [5], however the channel assignment maping the channel and the radio. 
International Journal of Wireless \& Mobile Networks (IJWMN) Vol. 9, No. 1, February 2017

The IEEE 802.11s uses the Mac layer routing protocol for multi-hop communication. In literature many routing metrics have been proposed to support the Multi-Channel Multi-Radios (MR-MC) architecture. Authors in [5] proposed a channel assignment method combined with routing over Multi-Radio Multi-Channel (MR-MC) Wireless Mesh Networks, this method improves throuhput and reduces end-to-end delay of network. Another method have been proposed in [6], which provides a new approach to the low throughput problem in multi-hop Wireless Mesh Network.

To improve the network capacity, we propose a new method based on channel assignment, combined with HWMP (Hybrid Wireless Mesh Protocol) protocol. This new method named ODCAM (On demand Assignment Method for channel diversity) proposes a new channel diversity mechanism used to select a new channel along the path between the source and the destination. The best path between the source and the destination is provided by HWMP using MWCETT an extension of the WCETT metric [7].

The ODCAM method aims to increase the network throughput and minimising the end-to-end delay in MR-MC architecture.

The rest of paper is organized as follows. Section II presents the related work, section III describes the proposed method ODCAM and section IV presents simulation results. Finally section V concludes the paper.

\section{RELATED WORK}

In order to increase the mesh network capacity, the WMN have evolved from single-radio singlechannel architecture to Multi-Channel Multi-Radios(MR-MC). The channel assignment find the optimal maping between the radio and the channel to improve the network performance [8].

In this section we study the channel assignment methodes, the channel diversity and the MAC layer routing protocols.

Several approaches for channel assignment have been proposed in literature [9]. Authors in [8], classified it in three categories: static, dynamic and hybrid approaches.

Static approach assigns one radio interface to one channel along the path. The limitation of this approach is it does not utilize the network resources effectively and furthermore it does not care about the factors that affect network performances [8]. Where dynamic approaches assigns one radio interface to one or many channels [9]. The principal challenges with the dynamic approach is how to choose the appropriate channel. Additionally authors in [8] proposed a hybrid approach based on static and dynamic appraoches. This approach aims to combine the advantages of static and dynamic approaches.

Recently, many researchers proposes several metric routing protocols for Wireless Mesh Networks (WMNs) [5, 6]. Some metrics are basesd on the channel diversity of the distant links along the path between the source and the destination. However authors in [5] propose a channel assignment combined with routing over Multi-Radio Multi-Channel (MR-MC) Wireless Mesh Networks. This method improve throuhput and reduce end-to-end delay of network, but this approach is implementing with layer three and based on DSR (Dynamic Source Routing) routing protocol [5]. 
Other method have been proposed in [6] based on AODV (Ad hoc On-demand Distance Vector) [10], it provides a new approach to the low throughput problem in multi-hop Wireless Mesh Network, but this solution is implemented in the network layer [6].

In [2], the IEEE 802.11s task group proposed the path selection protocol HWMP, based on AODV (Ad hoc On-demand Distance Vector)[10] but not uses a diversity routing metric [2].

To cope with the issuses stated above, we propose a new method to improve the capacity of Wireless Mesh Networks, which as based on channel asssignment and diversity mechanism. This new method is combined with MAC layer routing protocol HWMP, our method is detailed in the following section, however the first subsection presents the HWMP protocol.

\section{PROPOSED SOLUTION}

In this section we describe our proposed method named ODCAM (On Demand Channel Assignment Method for channel diversity). In order to improve the Wireless Mesh Networks capacity, the proposed solution aims to guarantee hop-by-hop channel diversity for HWMP protocol. This new method uses three components: channel assignment, channel diversity mechanism and HWMP routing protocol.

\subsection{MAC-layer Routing Protocol}

HWMP is a default MAC-layer protocol proposed by IEEE 802.11s task group [2], which combines two modes: on demand reactive mode based on AODV (Ad hoc On-demand Distance Vector) [10] and tree-based proactive mode [11]. First mode is used to construct a path between MPs, while in second mode a tree-based topology is created once an MP is configured as root. The two modes may be operated simultaneously, in this case which provides a best path is used. The HWMP uses the Airtime Link metric defined in [2] by:

$$
c_{a}=\left[O+B_{t}\right] \cdot\left[1 /\left(1-e_{f}\right)\right]
$$

- O : a overhead latency,

- $\quad B_{t}$ : test frame size in bytes,

- $\mathrm{r}$ : data rate in $\mathrm{Mb} / \mathrm{s}$,

- $\quad e_{f}$ : measured test frame error rate.

To communicate in the on-demand path discovery mode, the source MP include the destination MP address in PREQ (Path REQuest) frame and broadcasts it. Each intermediate node receives a PREQ, it verifies if it knows a path to destination, if is the case, this node sends a PREP (Path REPly) frame back to source. When source can set a DO (Destination Only) flag in the PREQ frame, only destination node is allowed to respond with PREP frame. When destination node receives a PREQ, it unicast PREP in reverse path to the source node.

The proactive tree-based mode, proposed two mechanisms: Proactive PREQ and Proactive RANN (Root Annoucement). In the first, when configured to work as a root MP, a node broadcasts a PREQ frame. This PREQ is sent periodiclly and every receiving MP broadcast the PREQ, which reaches all nodes in the MBSS. A node may sents a PREP frame back if it has data to send to the root node. 
The proactive RANN mechanism, intead of sending PREQs out a root node can flood the MBSS with RANN frames. To form a path to the root MP, the nodes send a PREQ frame to the root MP, the root node responds each PREQ with PREP, thus forming a forward path from each MP to the root MP.

ODCAM proposes a new Channel diversity mechanism and combines it with HWMP to select a new channel along the path between the source and the destination.

The MWCETT (Modified Weighted Cumulative Expected Transmission Time) an extension of WCETT metric determines the best path between the source and the destination.

This new solution is composed by two steps:

- Channel assignment and devercity mechanism,

- Implementation of the new method with HWMP.

These steps will be detailed in the next subsections.

\subsection{Channel assignment and Deversity mechanism}

The channel assignment is used to map the radio interface to the channel [8]. In order to increase the Wireless Mesh Network capaity, initaly each radio interface is assigned to one channel, until the intermediate node modifies it with the path selection process. In this case, the channel assignment method consists in :

- Including the channel selected in the request frame and sending it to the intermediate nodes,

- In order to guarantee the channel diversity, when the selected channel is equal to the channel of the previos hop then selecting a new channel.

Additionally, to store the selecting ans diversity channel information, each node along the path between the source and the destination uses a table named CDT (Channel Diversity Table) presented in Table 1.

Table 1: CDTi (CDT of the node i)

\begin{tabular}{|l|l|l|l|}
\hline Pid & CH & HC & MT \\
\hline
\end{tabular}

Where:

- Pid : the Path identifier,

- HC : the hop at the node i,

- $\mathrm{CH}$ : the selectd channel at the hop HC,

- MT : the metric value at the hop HC,

We define:

- $\mathrm{N}=\{\mathrm{N} 1, \mathrm{~N} 2, \ldots, \mathrm{Ni}, \ldots, \mathrm{Nm}\}$ a set of nodes in the path Pid,

- $\mathrm{C}=\{\mathrm{ch} 1, \mathrm{ch} 2, \ldots, \mathrm{chj}, \ldots \mathrm{chk}\}$ a set of channel,

$-\mathrm{R}=\{$ ri1, ri $2, \ldots$, rij,$\ldots$ rik $\}$ a set of radio interfaces. 
For each node Ni in path Pid we associete the value : $t v_{i}=\left(c h_{k}{ }^{i}, r i_{j}{ }_{j}, h c^{i}\right)$ and denotes li the link between the $\mathrm{Ni}$ and the node $\mathrm{Ni}-1$.

In this case, the node $\mathrm{Ni}$ assigns the intarface $r i_{j}{ }^{i}$ to the channel $c h_{k}{ }^{i}$, which is associetes to the link li at the hop $h c^{i}$.

A values $t v_{i}$ is equal to another $t v_{i+1}$ when:

$$
\left(c h_{k}{ }^{i}=c h_{k}{ }^{i+1}\right) \wedge\left[\left(r i_{j}{ }^{i}=r i_{j}{ }^{i+1}\right) \vee\left(r i_{j}{ }^{i} !=r i_{j}{ }^{i+1}\right)\right] \wedge\left(h c^{i} !=h c^{i+1}\right)
$$

The channel diversity mechanism is based on the comparison between $t v_{i}$ and $t v_{i+1}$. To select a new channel for link li at the hop hc, the node Ni runs a random function at the set of its available channels, switchs the active interface to the new selected channel and updates its CDT table. The following subsection presents the implementation of the new method with HWMP.

\subsection{Implementation of the new method with HWMP}

In this subsection, we focus on ODCAM method and the MAC-layer routing protcol HWMP. HWMP uses MWCETT (Modified Weighted Cumulative Expected Transmission Time) metric to find the best path between the source and the destination. Alongside the path between the source and the destination, each node uses a table CDT to store the channel assignment and diversity informations. Each record $l_{k}$ of CDT represents the selected channel informations at the path Pid:

- Pid : the Path identifier,

- HC : the hop at the node i,

- $\mathrm{CH}$ : the selectd channel at the hop HC,

- MT : the metric value at the hop HC,

To send or receive the channel assignment and diversity informations, the node uses the default MAC layer routing protocol HWMP. To send a flow fk to the destination node, the source node initiate the Path Request (PREQ) frame with channel informations (selected channel) and broadcast it. Each intermediate node checks if its tv is equal to the previos neighbor.

If the selected channel is equal to the channel of the previous hop, then node runs the slection channel function, stores the channel informations in CDT table, incrementes MWCETT metric, include the new channel in PREQ and forward it. After receiving PREQ, the destination node sends Path REPly (PREP) to the source node. After received PREP the source node setup the transfer of fk to the destination node.

To determine the best path between the source and the destination, ODCAM uses MWCETT an extension of WCETT metric [7] .

The WCETT (Weighted Cumulative Expected Transmission Time) as a path metric for routing in multi-radio multi-channel WMNs. To find paths with less intra-flow interference and channel diversity, the authors in [7] proposed WCETT, which is defined by: 


$$
W C E T T=(1-\beta) \sum_{i=1}^{i=n} \operatorname{ETT}_{i}+\beta \max _{k} X_{j}
$$

Where :

$-0<\beta<1$ : the tunable parameter,

- $\mathrm{Xj}$ : the summation of ETT of the links operating on the channel $\mathrm{j}$ in the path $\mathrm{p}$,

- $\mathrm{k}$ : the number of available channels,

- n: the length of the path $\mathrm{p}$.

And the MWCETT is defined by:

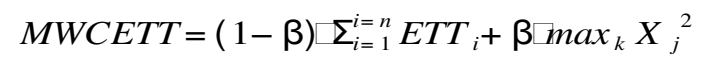

Where :

$X_{j}^{2}$ : the two hop summation of ETT of the links operating on the channel $\mathrm{j}$ in the path $\mathrm{p}$.

\section{SIMULATION AND RESULTS}

To evaluate the performance of ODCAM we use NS3 [12] simulator. In this simulation, we have assumed that 20 flows exists in the grid topology mesh network. The network size increases from 20 to 50 nodes and the nodes are operated in multi-radio multi- channel mode.

Table 2: Simulation parameters

\begin{tabular}{|l|l|}
\hline Parameter & Value \\
\hline Packet size & 512 bytes \\
\hline Data rate & $500 \mathrm{~kb} / \mathrm{s}$ \\
\hline Number of interface & 3 \\
\hline$\beta$ & 0.50 \\
\hline Link capacity & $3^{*} 54 \mathrm{Mb} / \mathrm{s}$ \\
\hline Number of flows & 20 \\
\hline Network size & $20-50$ \\
\hline
\end{tabular}

The simulation compares the hybrid channel assignment implementation with HWMP (HybHWMP) with the implementation of ODCAM with HWMP (ODCAM-HWMP). The two methods are compared by using two parameters: throughput and end-to-end delay.The average results are plotted in the graphs Figure 1 and Figure 2. The simulation parameters are presented in Table 2. 
International Journal of Wireless \& Mobile Networks (IJWMN) Vol. 9, No. 1, February 2017

The average throughput achieved using ODCAM-HWMP increase by $12.78 \%$ than Hyb-HWMP average throughput, Figure 1 shows the network throughput result.

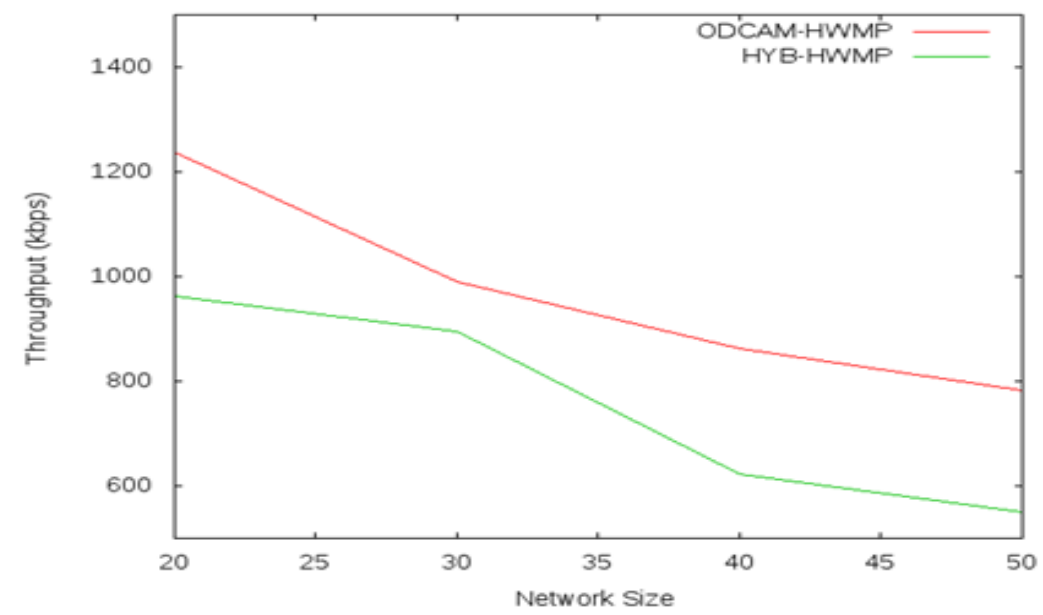

Figure 1: Average Throughput and Network size

Finally, the ODCAM-HWMP decreases the end-to-end delay by $1.1 \%$ than Hyb-HWMP, Figure 2 illustrates this result. When the network size is between 30 and 40 nodes, the value of this parameter given by ODCAM-HWMP is very similar to the same parameter achieving by HybHWMP. However, ODCAM-HWMP decreases this parameter, when the network size exceeds than 40 nodes.

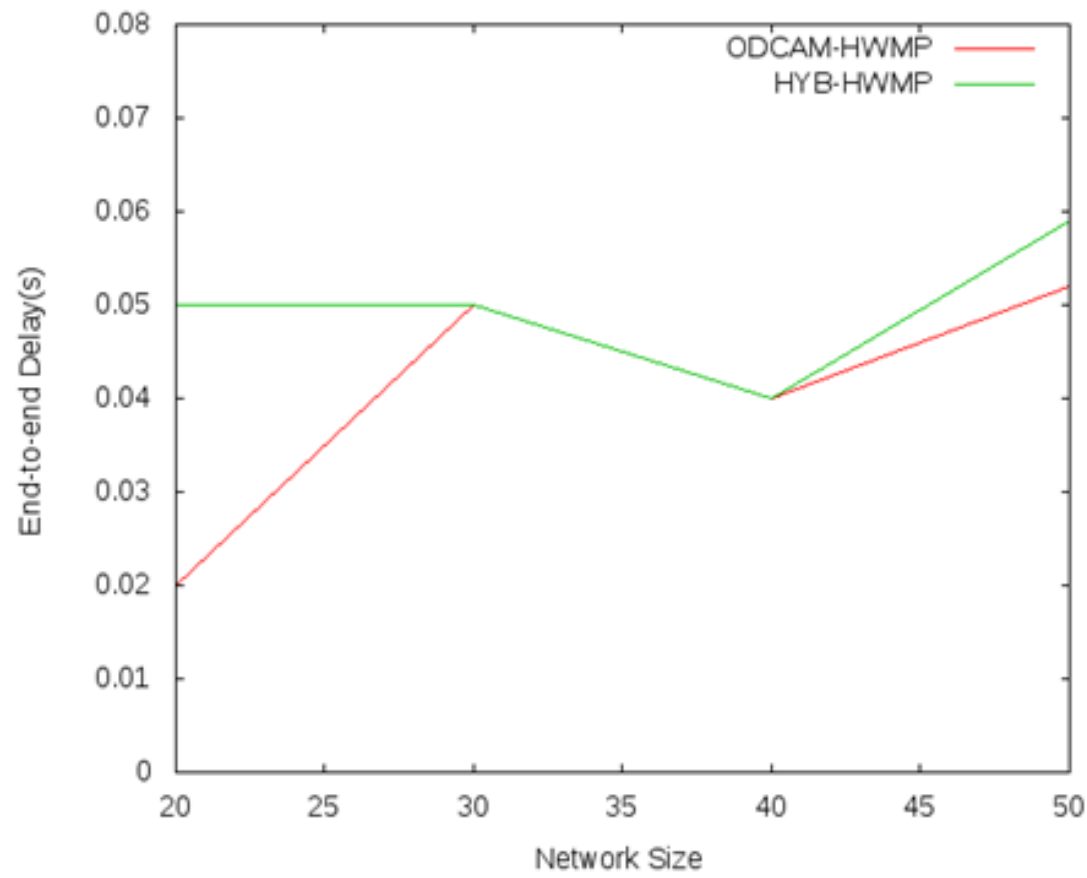

Figure 2: Average end-to-end delay and Network size 
International Journal of Wireless \& Mobile Networks (IJWMN) Vol. 9, No. 1, February 2017

\section{CONClusion}

In this article, we have proposed a new channel assignment method based on channel diversity. The ODCAM method provides a multi-hop channel diversity and uses MWCETT an extension of the WCETT metric with HWMP. We have shown the difference between our method (ODCAMHWMP), implemented with MAC-layer routing protocol HWMP and the implementation of the assignment Hybrid approach with HWMP (Hyb-HWMP). The simulation results show the ODCAM-HWMP performances compared with Hyb-HWMP: increasing the network throughput and decreases end-to-end delay.

\section{REFERENCES}

[1] IEEE standard for Information technology - Telecommunications and informations exchange between systems - local and metropolitan area networks - Specific requirements - Part 11: Wireless Medium Access Control (MAC) and Physical layer (PHY) Sepecifications, IEEE Standard, Approved 6 February 2012, sponsered by the LAN/MAN Standards commuttee of IEEE computer Society.

[2] IEEE 802.11s Task group, Draft Amendment to Standard for Information Technology Telecommunication and Information Exchange Between Systems - LAN/MAN Specific Requirements - Part 11: Wireless Medium Access Control (MAC) and physical layer (PHY) specification: Amendment: ESS Mesh Networking, IEEE P802.11s/D4.0, December 2009.

[3] A.Iera, A.Molinaro, S.Y.Paratore, G.Ruggeri, A.Zurzolo, "Making a mesh router/getway from a smartphone: is that a pratical solution?",Elsevier Ad hoc Netw.(2011),doi:10.1016/j.adhoc.2011.04.004.

[4] Sidi ould Cheikh, Abdelhak Gueroui, "Multi-hop Bandwidth Reservation in WMN-based IEEE 802.11s (MBRWMN)", IEEE ICCIT 2012.

[5] YUAN Fei-Fei, LI Xu,LIU Kai-ming, LIU Yuan-an, DU Xiao, and SHI Xin-rong "Distributed channel assignment combined with routing pver multi-radio multi-channel wireless mesh networks". ELSEVIER august 2012, (19):6-13.

[6] Michelle X.Gong, Scott F.Midkiff and Shiwen Mao "On demand routing and channel assignment mobiles ad hoc networks". ad Hoc Networks 7 (2009) 63-78.

[7] Skalli,H. et al.,"Channel assignment strategies for multiradio wireless mesh networks: issues and solutions". Communications Magazine, IEEE, 2007. 45(11): p. 86-95.

[8] A.Alzubir, K.Babakar, A.Yousif, A.Aboubieda. "State of the Art, channel Assignment Multi-Radio Multi-channel in Wireless Mesh Network". International Journal of computer Applications (09758887) Volume 37-No.4, January 2012.

[9] W.Si, S.Selvakennedy, and A.Y. Zomaya, "An overview of channel assignment methods for multiradio multi- channel wireless mesh networks". Journal of Parallel and Distributed Computing, 2010. 70(5): p. 505-524.

[10] C.Parkins, E.Belding-Royer,S.Das, "Ad Hoc on-demand-distance Vector(AODV) routing", IETF RFC 3561, 2003.

[11] Cheikh Sarr, Isabelle Guerin Lassous. "Estimating Average End-to-Ende Delays in IEEE 802.11 Multi-hop Wireless Networks". researh report July 2007.

[12] "NS-3", Network Simulator. Available: http://www.nsnam.org. 